\title{
Age and disease-related structural changes in the retinal pigment epithelium
}

\author{
Vera L Bonilha \\ Cole Eye Institute, The Cleveland \\ Clinic, Cleveland, OH, USA
}

Correspondence: Vera L Bonilha

Cole Eye Institute (i3I), The Cleveland

Clinic, 9500 Euclid Avenue, Cleveland, OH

44195, USA

Tel +I 2164457690

Fax +I 2164453670

Email bonilhav@ccf.org

\begin{abstract}
As the retinal pigment epithelium (RPE) ages, a number of structural changes occur, including loss of melanin granules, increase in the density of residual bodies, accumulation of lipofuscin, accumulation of basal deposits on or within Bruch's membrane, formation of drusen (between the basal lamina of the RPE and the inner collagenous layer of Bruch's membrane), thickening of Bruch's membrane, microvilli atrophy and disorganization of the basal infoldings. Although these changes are well known, the basic mechanisms involved in them are frequently poorly understood. These age-related changes progress slowly and vary in severity in different individuals. These changes are also found in age-related macular degeneration (AMD), a late onset disease that severely impacts the RPE, but they are much more pronounced than during normal aging. However, the changes in AMD lead to severe loss of vision. Given the many supporting functions which the RPE serves for the retina, it is important to decipher the age-related changes in this epithelium in order to understand age-related changes in vision.

Keywords: retinal pigment epithelium, aging, age-related macular degeneration (AMD), ocular disorders, retinal disease
\end{abstract}

\section{Age-related changes in the RPE}

The retinal pigment epithelium (RPE) performs highly specialized metabolic and transport functions essential for homeostasis of the neural retina (Bok 1993). These include phagocytosis of photoreceptor-shed outer segments, transport of nutrients into and removal of waste products from photoreceptor cells and retinoid transport and regeneration. The RPE is a low cuboidal epithelium containing very long thin and sheet-like microvilli on its apical surface that project into the interphotoreceptor matrix where they interact with the tips of the rod and cone photoreceptor outer segments (Bok 1993). The apical surface of RPE cells supports and carries out the diurnal phagocytic removal of spent photoreceptor tips. One RPE cell supports 30-50 photoreceptors, which shed daily $\sim 5 \%$ of their outer segment mass (Zinn and Benjamin-Henkind 1979). The basal surface of RPE cells displays highly convoluted basal infoldings that attach to a specialized Bruch's basement membrane, an acellular layer separating the RPE from the choriocapillaris. The RPE's basal surface participates in extensive metabolic exchanges with the blood vessels in the underlying choriocapillaris.

An accumulation of discrete but pronounced structural changes occurs in aging eyes. In the aged retina, an overall thinning is apparent, due to loss of neurons from all the neuronal cells and also shortening of photoreceptor cells. The RPE specifically is known to undergo several structural changes, including loss of melanin granules, increase in the number of residual bodies, accumulation of the age pigment lipofuscin, accumulation of basal deposits on or within Bruch's membrane (BM), formation of drusen (between the basal lamina of the RPE and the inner collagenous layer of BM), thickening of BM, RPE microvilli atrophy and disorganization of basal infoldings (Boulton and Dayhaw-Barker 2001). Some of these changes are shown in Figure 1 

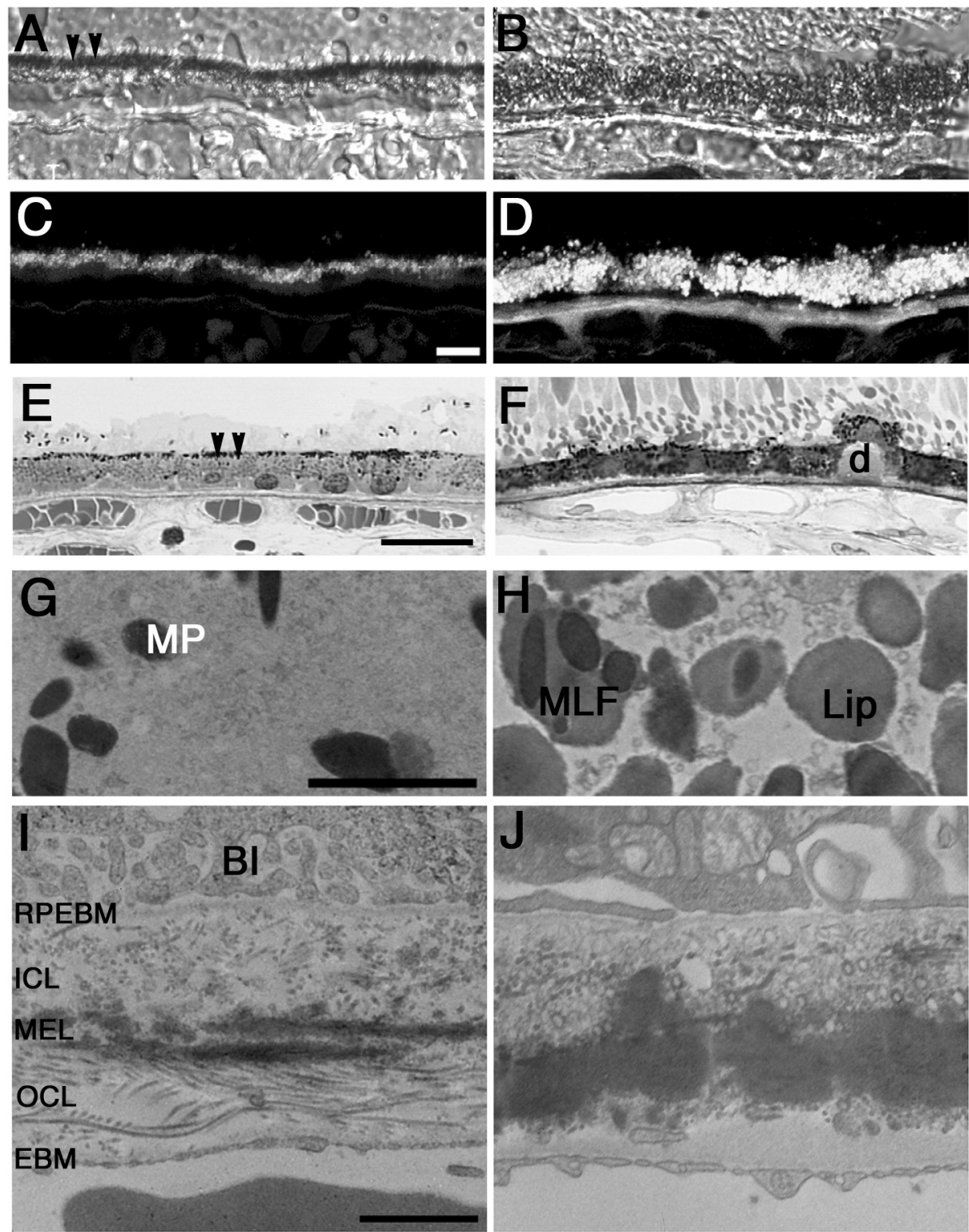

Figure I Age-related changes in human RPE. Observation of the structural differences in RPE from young ( 23 year-old, A, C, E, G, I) and aged (75 and 88 year-old, B, D, F, $\mathbf{H}, \mathbf{J}$ ) human donors. Aged RPE from human donors displays loss of melanin granules (MP, arrowheads in A, E) and accumulation of the age pigment lipofuscin (Lip) (B, D), as observed by the presence of increased autofluorescent granules when observed on epifluorescence in the green channel (FITC filter: excitation $495 \mathrm{~nm} / \mathrm{emission} 5 \mathrm{I} 9 \mathrm{~nm}$ ) in aged RPE (D) when compared to young RPE (C).Additional observation of the aged RPE displayed formation of drusen (D) (between the basal lamina of the RPE and the inner collagenous layer of Bruch's membrane) (F), thickening of Bruch's membrane and basal infoldings disorganization (J) when processed and analyzed by electron microscopy. In addition, in the aged RPE cells melanin granules are frequently seen in association with lipofuscin (melanolipofuscin, MLF) granules (H). Young RPE displays melanin pigments on their apical surface (A, E, G) while aged RPE contains mostly lipofuscin granules (B, H). Differential interference contrast microscopy images (A, B). Semi-thin epon sections stained with toluidine blue of young (E) and aged RPE (F) examined in bright field.

Abbreviations: BI, basal infoldings; RPEBM, RPE basement membrane; ICL, inner collagenous layer; MEL, middle elastic layer; OCL, outer collagenous layer; EBM, choroidal endothelial cell basement membrane; Bars: (A to D), $10 \mu \mathrm{m} ;(\mathbf{E}, \mathbf{F}), 200 \mu \mathrm{m} ;(\mathbf{G}, \mathbf{H}), 2 \mu \mathrm{m}$; (I, J), I $\mu \mathrm{m}$.

(B, D, F, H, J) and they will be discussed in detail in the following text. The RPE aging changes progress slowly and are of varying severity in different eyes.

The RPE contains two kinds of pigment, namely lipofuscin and melanin. Melanin is an insoluble high molecular weight polymer derived from the enzymatic oxidation of tyrosine and dihydroxyphenylalanine, linked to proteins and contained in membrane-limited granules in the RPE melanosomes. Recently a comprehensive determination of the protein composition of melanosomes isolated from human melanoma cells was reported using proteomics (Chi et al 2006). The identified proteins included 16 homologs to mouse coat color genes, many associated with human pigmentary diseases, pigment epithelium-derived factor (PEDF) and SLC24A5 (sodium/potassium/calcium exchanger 5, NCKX5). However, these melanosomes may be different 
from the ones present in the RPE. RPE melanin originates from neural ectoderm, whereas the one in melanocytes originates from neural crest (Feeney 1978). In aged RPE cells melanin granules are frequently seen in association with lysosomes (melanolysosomes, MLL) and lipofuscin granules (melanolipofuscin, MLF; Figure 1H), which suggests that any protein bound to melanin may be degraded. In addition, melanosomes may undergo photobleaching with aging, which can diminish the antioxidant efficiency of melanin (Sarna et al 2003). Altogether, these observations suggest that changes in melanin granules possibly contribute to some of the senile changes evident in the RPE. A recent manuscript observed the accumulation of MLF in human RPE from different decades of life and assessed their phototoxicity to RPE cultures in vitro. The analysis of the composition of MLF granules suggested that, in contrast to lipofuscin, they do not contain photoreceptor-specific proteins. The authors suggest that MLF may not originate from photoreceptor outer segments phagocytosis but that MLF accumulates as a result of the melanosomal autophagocytosis of RPE cells (Warburton et al 2006).

Accumulation of secondary lysosomes and residual bodies containing lipofuscin, known as dense bodies, has been observed in post-mitotic and intermitotic cells during aging (Schmucker and Sachs 2002; Morales et al 2004; Kubasik-Juraniec et al 2004). The general consensus is that the accumulation of these dense bodies represents lysosomal aging and is a universal index of cellular senescence (Schmucker and Sachs 2002; Terman et al 2007). It has been well established that the RPE has an extremely active lysosomal system capable of degrading thousands of phagocytosed outer segment disks per day (Young 1971; Zinn and Benjamin-Henkind 1979). The aged RPE accumulates indigested residues of this phagocytic process as residual bodies (Feeney-Burns et al 1987).

Lipofuscin pigment has been described as intracellular yellow-brown autofluorescent granules exhibiting sudanophilic, osmiophilic, argyrophilic and periodic acid-Schiffpositive and acid-fast staining characteristics (Feeney 1978). Lipofuscin is a heterogeneous material composed of a mixture of lipids and different fluorescent compounds, the main fluorophore of which has been identified as the pyridinium bis-retinoid, $N$-retinylidene- $N$-retinylethanolamine (A2E), a derivative of vitamin A. RPE lipofuscin is unique because it originates mainly from the phagocytosed photoreceptor outer segments as was demonstrated in early studies. For instance, analysis of the chemical composition of RPE cells revealed that it is different from the photoreceptor outer segments (Berman et al 1974). In addition, investigations undertaken on the Royal College of Surgeons (RCS) rats showed that in this strain, which fails to phagocytose shed outer segments, lipofuscin is significantly diminished (Katz et al 1986; Eldred and Lasky 1993). Moreover, the accumulation of autofluorescent debris was observed in a transgenic mouse line expressing a mutated form of cathepsin D that is enzymatically inactive, thereby impairing the processing of phagocytosed photoreceptor outer segments by the RPE cells (Rakoczy et al 2002). A recent study established the presence of extragranular material present in preparations of lipofuscin routinely isolated by sucrose density gradient centrifugation. In this study, the lipofuscin granules were isolated and further purified by digestion of the extragranular material with proteinase K or by wash with SDS detergent. Raw and purified granules were tested for their protein content. The results demonstrated that: debris-free granules contain little or no protein; the protein associated with lipofuscin granules is essentially all extra-granular and appears to be significantly modified by posttranslational modifications (Renganathan et al 2007). Lipofuscin granules first appear in the basal portions of RPE cells of young eyes (1st decade, Figure 1C), whereas in older eyes (9th decade, Figure 1D), lipofuscin granules form into clumps and fill the entire RPE cell cytoplasm (Wing et al 1978). It is suggested that the accumulation of lipofuscin in aged RPE is connected to RPE functional degeneration either by "clogging" of the cytoplasm or by increased oxidative stress in the cell. Support for the first mechanism (clogging of the cytoplasm) comes from a recent study, which implanted glycoxidized microspheres (Glycox-MS) as imitation for lipofuscin into the subretinal space of 10-12 week-old rabbits. Observations were carried out from 1 to 16 weeks after subretinal implantation. GlycoxMS stagnated for a prolonged period in the cytoplasm of RPE cells and eyes implanted with glycox-MS produced drusenlike deposits at a significantly higher frequency (Yasukawa et al 2007). Support for the second mechanism (increased oxidative stress in the RPE cells) comes from observations that lipofuscin is a photoinducible generator of superoxide anion, singlet oxygen and hydrogen peroxide (Boulton et al 1993; Gaillard et al 1995; Rozanowska et al 1995, 1998). Thus, visible-light irradiation (400-1100 nm) of lipofuscin granules results in extra-granular oxidation of lipids and inactivation of lysosomal and antioxidant enzymes (Wassel et al 1999). In addition, it was shown that A2E has phototoxic and detergent properties and is capable of inducing disintegration of membrane-bound organelles in RPE cultures. Finally, lipofuscin can also interfere with the antioxidant properties of 
melanin (Boulton et al 1993; Rozanowska et al 1995; Schutt et al 2001, 2002; Wang et al 2006). The aged RPE displays increased intracellular accumulation of the blue-shifted autofluorescence lipofuscin granules, which coincides with the depletion of melanin pigments (Feeney-Burns 1984; Han et al 2007). RPE lipofuscin granules exhibit a broad band emission spectrum with a peak at $600 \mathrm{~nm}$ and subsidiary shoulders located at 470 and $550 \mathrm{~nm}$ when excited at $364 \mathrm{~nm}$; a 680 peak appears with increasing age (Boulton et al 1990).

Bruch's membrane (BM) is a pentalaminar structure composed of the RPE basement membrane, inner collagenous layer, middle elastic layer, outer collagenous layer, and the choroidal endothelial cell basement membrane (Hogan and Alvarado 1967). This acellular extracellular meshwork found between the RPE and the choroid, which is $2-4 \mu \mathrm{m}$ thick is known to undergo increased thickening (Figure 1J), chemical reconfiguration of both proteins and lipids, and debris accumulation during aging (Pauleikhoff et al 1990; Okubo et al 1999; Zarbin 2004). The aged BM displays an exponential increase in phospholipids, triglycerides, fatty acids, and free cholesterol content (Sheraidah et al 1993). Protein reconfiguration in the form of post-translational modifications has been reported in the BM. Immunoreactivity to some of the advanced glycation end product (AGE) adducts increases in the aged BM (Farboud et al 1999; Handa et al 1999). Although the precise contribution of AGEs to the retinal pathology remains to be elucidated, AGEs are recognized as important initiators of age-related dysfunction, inasmuch as they are known to cause protein cross-linking, reduced solubility, enzymatic dysfunction, and loss of receptor recognition (Baynes 2001). A recent study combined both Raman microscopy and specific chemical quantification to assess defined AGE adducts and quantify AGE-related spectral alterations in aged BM of postmortem eyes (Glenn et al 2007). The analysis showed that the AGEs pentosidine, carboxymethyllysine (CML), and carboxyethyllysine (CEL) occurred at significantly higher levels in BMCh with age (Glenn et al 2007). In addition, several recent studies demonstrated that tissue metalloproteinase inhibitor 3 (TIMP-3), vitronectin, annexins, crystallins, clathrin and adaptin proteins were crosslinked as evidenced from western blots that showed the presence of these proteins at several regions of the gel (Nakata et al 2005; Rayborn et al 2006; Bando et al 2007).

In addition, BM is under constant cycles of pressureinduced stress as a result of the choroidal flow oscillating with the cardiac rhythm. The mechanical properties of BM are critical determinants of its physiology. Specifically, the elastic properties of BM will determine its ability to sustain potentially damaging stress and strain perturbations. Recently the mechanical properties of isolated human BM were investigated and related to aging. This study demonstrated that the elasticity of human BM-Ch complex decreased linearly with aging after the age of 21 with an approximate reduction of $1 \%$ per year. On the other hand, the recoil capacity of Bruch's membrane-choroid was not affected by aging (Ugarte et al 2006).

Drusen are debris-like deposits that accumulate below the RPE along BM (Figure 1F). Clinically, they are characterized by the terms "hard" and "soft" according to their size and their appearance in fluorescein angiography. Hard drusen are small, hard, round and have well defined borders (Marshall et al 1998). Hard drusen occur in 80\% of postmortem eyes, and are usually small, they are hyperfluorescent on fluorescein angiography, a characteristic that may be related to the fact that they are enriched in phospholipids (Bird and Marshall 1986; Pauleikhoff et al 1992; Arnold et al 1997). On the other hand, soft drusen are extensive, diffuse, large deposits, which have borders not sharply defined, and rarely occur before the age of 55 (Garner et al 1994; Marshall et al 1998). Soft drusen are hypofluorescent in fluorescein angiography and display a high content of neutral fats (Pauleikhoff et al 1992; Arnold et al 1997), vesicles, membranous debris, and wide-spaced collagen. Generally, hard drusen do not lead to loss of vision, but soft drusen are considered contributors to the pathology of age-related macular degeneration (AMD). Soft drusen deposition in the macula precedes visual loss; it defines the early stages of AMD together with pigmentary changes of the RPE. Deposition of soft drusen in the macula is considered the precursor lesion that leads to the development of geographic atrophy (dry AMD) and choroidal neovascularization (wet AMD), which are the late forms of AMD. The different types of AMD will be discussed in detail in the following text.

A recent proteomic study carried out on isolated drusen from both AMD and normal donors found up to $65 \%$ of the proteins identified common to both donor types. TIMP-3, clusterin, vitronectin, and serum albumin were the most common proteins observed in normal donor drusen, whereas crystallin was detected more frequently in AMD donor drusen. In addition, protein from both normal and AMD donors such as vitronectin, TIMP-3, clusterin, complement C9, lysosyme $\mathrm{C}$, serum amyloid $\mathrm{P}$, and apolipoprotein $\mathrm{E}$ migrated in multiple mass ranges from the top to the bottom of the gel, suggesting the presence of covalent crosslinks (Crabb et al 2002). 
Recent work from several groups also suggests that local inflammation plays a role in drusen formation in a process analogous to that which occurs in other age-related diseases such as Alzheimer's disease and atherosclerosis, in which there is an accumulation of extracellular plaques and deposits causing a local chronic inflammatory response which in turn exacerbates the effects of the primary stimuli (Hageman et al 2001; Johnson et al 2001; Anderson et al 2002). This hypothesis is supported by evidence revealing the localization of several proteins involved in the immune system such as immunoglobulins; components of complement cascade (such as C5b-9 complex, complement factor F); MHC class II antigens; cell-associated molecules, including HLA-DR and specific CD antigens (Mullins et al 2000; Hageman et al 2001; Johnson et al 2001; Anderson et al 2002).

Few studies have demonstrated age-related effects on RPE microvilli. A finding common to all of them was the shortening of the RPE microvilli (Katz and Robison 1984; Lai and Rana 1986; Weisse 1995). Previously, we have been able to isolate intact RPE microvilli from mice (Bonilha et al 2004) and characterize its content using proteomics. Several of the identified proteins in the microvilli fraction are antioxidant enzymes and have been shown to undergo specific modulation during aging. These include lactate dehydrogenase, glutathione S-transferase, peroxiredoxin, ceruloplasmin, and superoxide dismutase. Our data are supported by several reports, which identified the presence of antioxidative enzymes in the microvilli of kidney (Davies et al 1993; Muse et al 1994), respiratory tract epithelium (Coursin et al 1992), and intestine (Davis et al 1989), among others. Oxidation is a very important mechanism in aging (Kohen and Nyska 2002; Balazy and Nigam 2003; Van Remmen et al 2003; Kregel and Zhang 2007). RPE apical microvilli shortening is also expected to affect several of the key functions carried out by the apical surface. Examples of these include phagocytosis of shed photoreceptor outer segments through the receptors $\alpha \mathrm{v} \beta 5$ vitronectin receptor and the Mer tyrosine kinase (MerTK) receptor protein; apical transport involving transporters such as $\mathrm{Na}, \mathrm{K}-\mathrm{ATPase}$, the glucose transporter (Glut-1), monocarboxylate transporter 1 (MCT1), basigin, the Kir7.1 K+ channel, chloride intracellular channel 6 , carbonic anhydrase XIV, among others; and visual cycle function through the involvement of CRALBP, RPE65, IRBP, and CRBP (Rayborn et al 2005). These changes could alter the retinal metabolic equilibrium and accelerate degenerative processes in the aging retina. Our ongoing research aims to identify a protein profile that is uniquely present in aged RPE cells. Our findings will lead to future studies on the functional consequence of these proteins and to a more complete understanding of the pathogenesis of AMD.

\section{Animal models in RPE aging studies}

A good animal model should reduplicate biochemical, morphological, and molecular changes shown in humans during aging. Much of our understanding of the biological changes that occur with aging has come from studies using rodents. Similarities in the physiology and cell biology of aging in humans and rodents make rodents a valuable model with which to test therapeutic interventions for aging, and they are small enough to allow for the use of statistically robust sample sizes. There are several rodent models to choose from. The National Institute on Aging (NIA) supports many resources to facilitate the use of animal models for biogerontological research, including aged rodent colonies, the aged rodent tissue bank, and tissue arrays from aged rodents (Nadon 2006). One of the rodent models available for aging studies is the rat $\mathrm{F}_{1} \mathrm{~F} 344 / \mathrm{BN}$ hybrid. The aged (24-25 month-old) F344/BN rat displays several of the RPE age-related changes described above; specifically, BM thickening, lipofuscin accumulation, accumulation of residual bodies, decrease in RPE density and microvilli atrophy when compared to young (3-4 month-old) rats (Figure 2).

\section{Age-related changes in RPE density}

Numerous studies have been undertaken to determine changes in RPE density with age. However, previous studies yielded contradictory results: Some found that RPE density increased with age (Tso and Friedman 1968; Harman et al 1997; Leung et al 2004). Others found that it decreased (Streeten 1969; Gao and Hollyfield 1992; Watzke et al 1993); while yet another study found that it did not change with age at all (Dorey et al 1989). The discrepancies among these studies can be explained by the number of eyes analyzed and by the nature of the analyses which were carried out. Use of cross-sections allows analysis of a restricted number of cells, whereas whole-mount preparations allows analysis of the whole population of cells in the tissue. One study (Gao and Hollyfield 1992) investigated this issue analyzing eyes from donors from the 2 nd to the 9 th decade. Tissue fragments were obtained and analyzed as whole-mounts from the fovea and the retinal equator. Observations suggested that foveal RPE is denser, with cells smaller and more homogeneous, independent of the age of the donor, as shown in Figure 3A and C. Linear regression of the obtained data yielded a significant negative slope of RPE density in the retinal equator, suggesting a uniform rate of equatorial RPE loss during aging. 


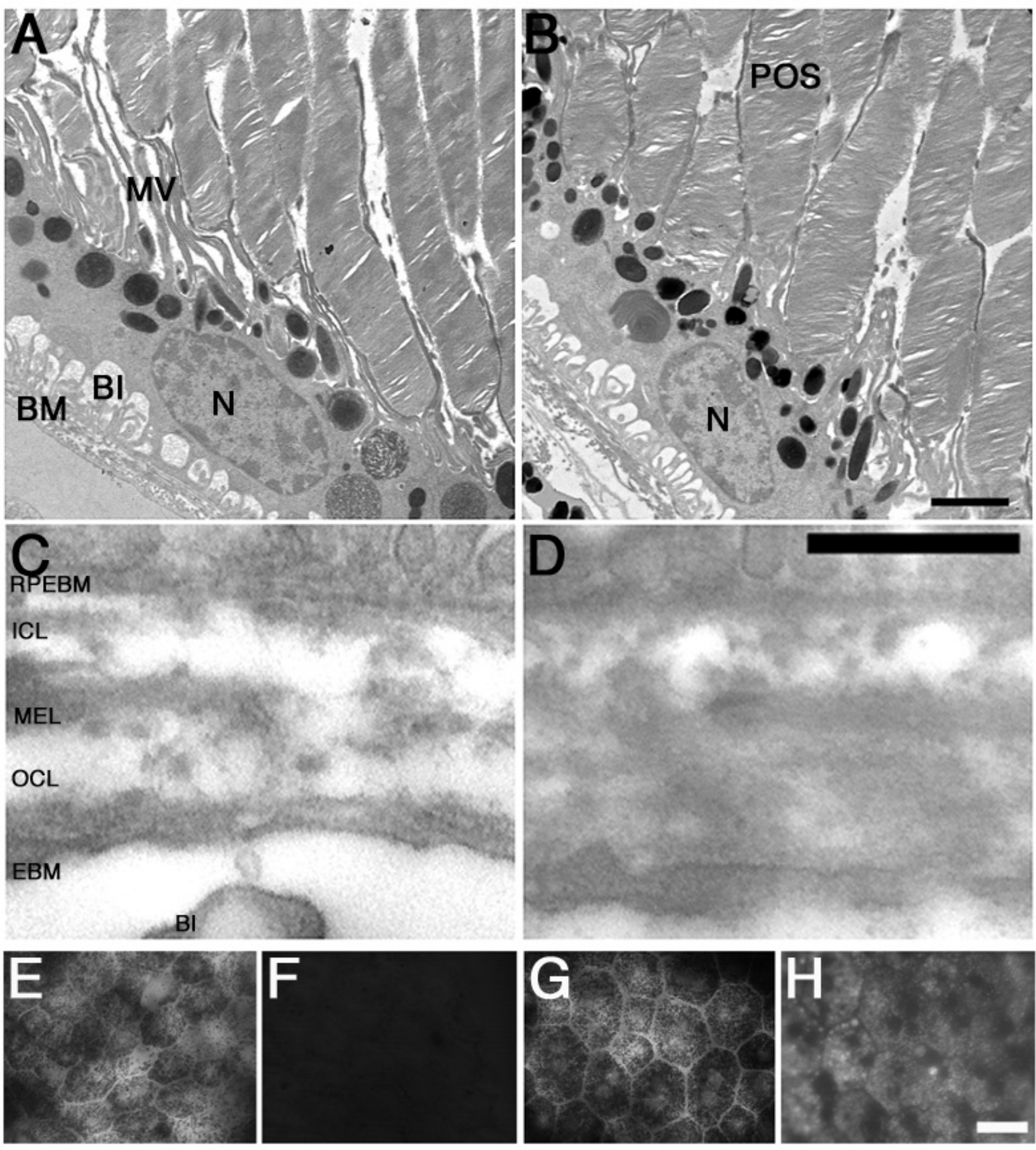

Figure 2 Age-related changes in FI F344BN hybrid rat RPE. Observation of young (3-4 month-old, A) and aged (24-25 month-old, B) FI F344BN hybrid rats reveals several of the RPE age-related changes previously described. These include: Bruch's membrane thickening (D), accumulation of residual bodies, and microvilli atrophy (B). In addition, bright-field analysis of aged RPE whole-mounts reveals decrease in RPE density (G) while epifluorescence in the green channel (FITC filter: excitation $495 \mathrm{~nm} / \mathrm{emission} 519 \mathrm{~nm}$ ) reveals increased lipofuscin accumulation $(\mathbf{H})$ when compared to the young RPE cells (E and $\mathbf{F})$. A-D. Transmission electron microscopy.

Abbreviations: $\mathrm{Bl}$, basal infoldings; MV, microvilli; POS, photorecptor outer segments; RPEBM, RPE basement membrane; ICL, inner collagenous layer; MEL, middle elastic layer; OCL, outer collagenous layer; EBM, choroidal endothelial cell basement membrane; Bars: (A and B), I $\mu \mathrm{m}$; (C and D), $2 \mu \mathrm{m}$; and (C to F), $200 \mu \mathrm{m}$. 

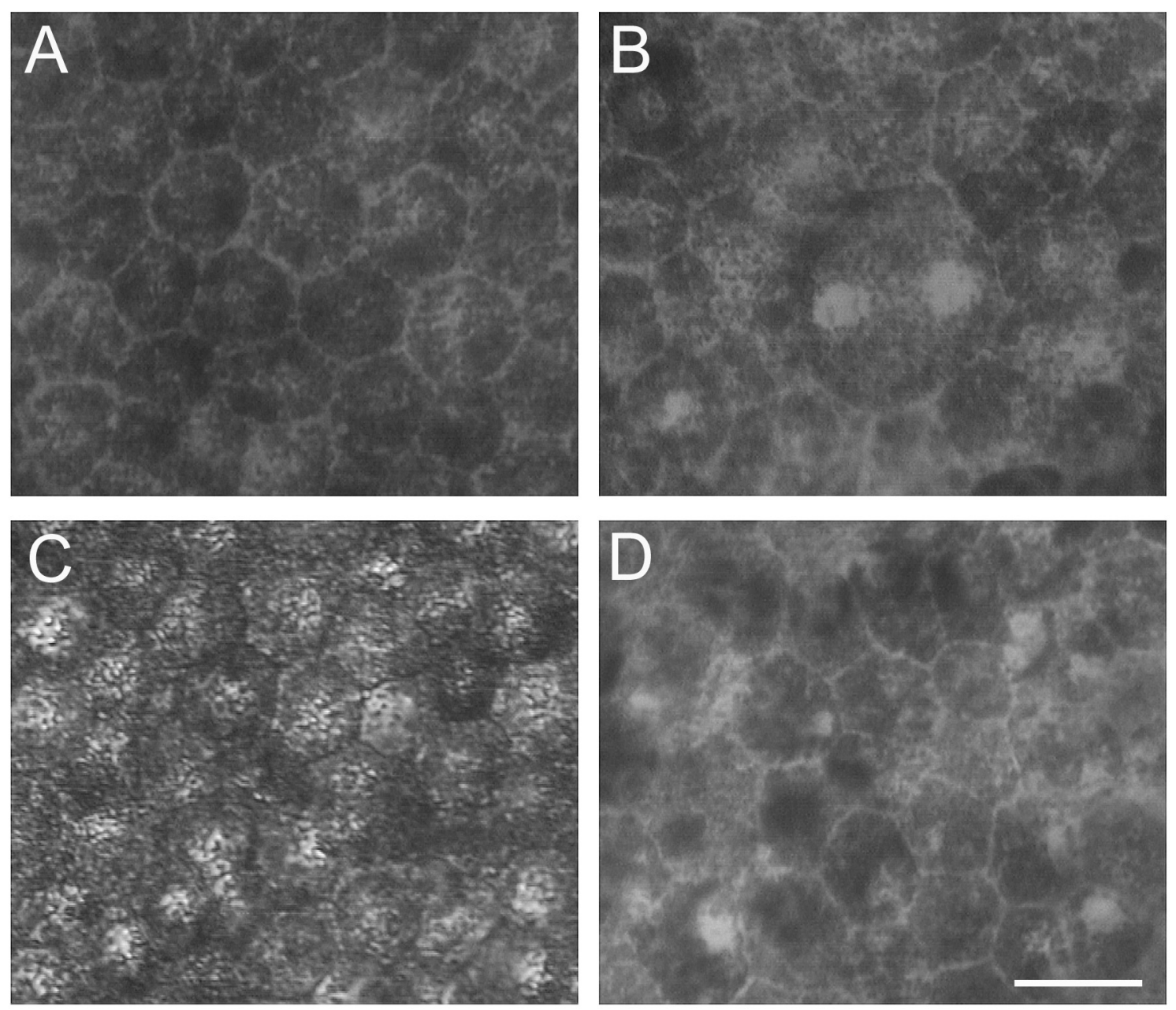

Figure 3 Age-related changes in RPE density. Bright-field micrographs of RPE whole- mounts from both young (A and $\mathbf{B})$ and aged (C and $\mathbf{D})$ donor eyes. Observations were carried out both in the fovea ( $\mathbf{A}$ and $\mathbf{C}$ ) and periphery (B and $\mathbf{D})$ of the eyes. Foveal RPE cells are smaller and more homogeneous than the peripheral RPE cells independent of the age of the donor. Bas, $200 \mu \mathrm{m}$.

On the other hand, foveal RPE density was relatively stable from the 2nd through the 9th decades, with no significant decrease in cell density (Gao and Hollyfield 1992).

Another study investigated the age-related changes in RPE in an even larger number of eyes from donors from the 2nd to the 9th decade (Panda-Jones et al 1996). Using a $3 \mathrm{~mm}$ trephine the authors collected RPE/retina/choroid in the fovea and in the superior, inferior, temporal, and nasal meridians in 6 rings that were arranged concentrically around the fovea. These samples were also analyzed as whole-mount preparations. As in the previous study, the authors concluded that RPE density at the foveal center was the highest and that it decreased significantly from the fovea to the mid-retinal periphery. In the periphery, RPE density was the highest in the nasal region. The age-related loss was most marked in the fovea and the mid periphery. The authors determined that RPE cell density in the fovea decreased significantly by about $0.3 \%$ per year with increasing age.
The elderly suffer from loss of visual acuity (Weale 1975; Del Viva and Agostini 2007), color perception (Ohta and Kato 1975; Page and Crognale 2005), and dark-adaptation sensitivity (McFarland et al 1960; Werner 2005). These conditions are probably associated with age-related death of RPE and photoreceptors. It is important to understand the mechanisms involved in these cell deaths. One study specifically addressed this issue by analyzing agerelated RPE apoptosis through terminal deoxynucleotidyl transferase-mediated dUTP nick end (TUNEL)-labeling in whole-mount preparations of eyes divided into 4 concentric regions centered on the fovea. Overall, analysis showed that TUNEL-positive RPE cells were uncommon. There was a significant positive correlation between the donor's age and the number of apoptotic cells. Analysis of the individual regions revealed that within zone $1(0-1.5 \mathrm{~mm}$ radius $)$ the proportion of apoptotic RPE cells started to increase in the 6th decade. There was also an age-dependent increase in 
apoptosis within zone $2(1.5-3.0 \mathrm{~mm}$ radius, at a much lower number) (Del Priore et al 2002).

\section{RPE changes and loss in AMD}

AMD is the most common cause of irreversible blindness in the elderly population in industrialized countries (Leibowitz et al 1980; Klein et al 1995; Kelin et al 2004). Although aging is an important event that contributes to the pathogenesis of AMD, it does not directly lead to the occurrence of AMD (Sarks 1976; Young 1987). AMD occurs in two forms: neovascular or exudative (wet) and atrophic (dry) AMD. Neovascular AMD is characterized by abnormal growth of capillaries from the choroid into the Bruch's membrane and RPE and by subsequent exudation of fluid, lipid, and blood. It results ultimately in a disciform scar in the macula and is responsible for severe, sudden visual loss (Holloway and Verhoeff 1929; Verhoeff and Grossman 1937). Atrophic AMD, also known as geographic atrophy (GA) is characterized by a progressing course leading to degeneration of RPE and photoreceptors. Studies have shown that the atrophy initially tends to develop in the perifoveal area, while the fovea may be spared until later during the clinical course (Sarks et al 1988; Sunness 1999). GA is characterized by a loss of the outer neurosensory retina, the RPE, and the choriocapillaris (Figure 4). The primary dysfunction and cell death of the RPE cells is thought to occur initially, followed by collateral loss of neighboring photoreceptor cells and choriocapillaris (Sarks et al 1988; Roth et al 2004). Previous studies have shown that photoreceptor apoptosis is involved in AMD pathology (Green and Enger 1993; Xu et al 1996). Recently, another study quantified the number of TUNEL-positive cells in each retinal layer in cryosections of AMD and control eyes. The authors showed that maculas with AMD had a statistically significant increase in TUNEL-positive RPE cells compared with the control ones. In the GA eyes, TUNEL-positive rods and RPE nuclei were present near the edges of RPE atrophy (Dunaief et al 2002). Additional data also suggest that A2E, a lipofuscin component, induces apoptosis in RPE cultures (Suter et al 2000). Finally, apoptosis was also observed in surgically excised choroidal neovascular (CNV) membranes from AMD-affected eyes (Hinton et al 1998).

Clinical features common in both types of AMD include the presence of drusen and hypo- and hyperpigmentation of the RPE. Histological features of RPE in AMD include accumulation of lipofuscin, formation of drusen and of basal deposits in the BM, and alteration in the BM extracellular matrix (Hogan 1972; Sarks 1976; Young 1987; Green and Enger 1993; Roth et al 2004; Nowak 2006). As mentioned above, these features are also observed, with lower intensity, in the aging RPE. However, the changes in AMD lead to severe loss of vision.

AMD is a multifactor disease with genetic components (Klaver and Allikmets 2003; Gold et al 2006; Hageman et al 2006; Scholl et al 2007). However, exogenous factors such as light exposure, a high fat diet, high blood pressure, and smoking (Leibowitz et al 1980; Cruickshanks et al 1993; Cousins et al 2002) are known to modulate its pathogenesis. In addition, abnormal regulation of the complement system, likely caused by the $\mathrm{Y} 402 \mathrm{H}$ polymorphism in the complement factor $\mathrm{H}$ gene on 1q, is a recognized risk factor for $\mathrm{AMD}$, as is the A69S variant in the poorly characterized LOC387715 gene and the serine protease HTRA1 in multiple populations (Edwards et al 2005; Haines et al 2005; Jakobsdottir et al 2005; Klein et al 2005; Rivera et al 2005; Dewan et al 2006;

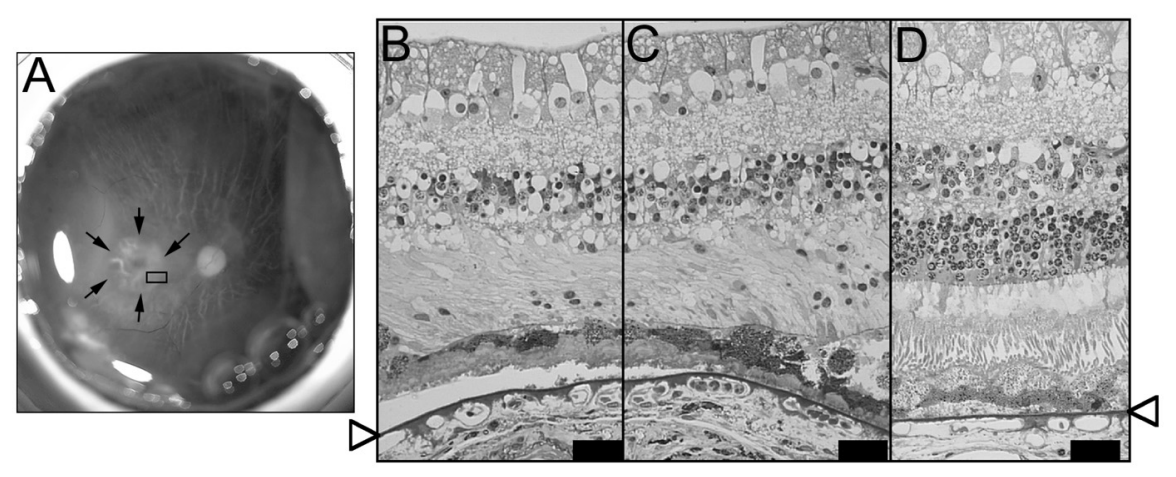

Figure 4 RPE cell loss in geographic atrophy. Gross photomicrography of a postmortem eye from an AMD donor with geographic atrophy (GA) (A).Arrows indicate the edges of GA; inset indicates the region cut and processed for transmission electron microscopy. Semi-thin epon sections stained with toluidine blue of this region demonstrates extensive RPE, photoreceptors and choroids atrophy in the GA region (B) while the edge of GA displays more RPE cells and the presence of some photoreceptor outer segments (C). In the region outside of the GA the RPE layer is continuous and the photoreceptors' inner and outer segments can be observed (D). Arrowheads point to Bruch's membrane. Debris accumulation is observed underneath the RPE cells in all regions observed. Bars $200 \mu \mathrm{m}$. 
Yang et al 2006). On the other hand, polymorphisms in the factor B and complement component 2 are associated with decreased susceptibility to AMD (Gold et al 2006; Spencer et al 2007). Previous studies had identified the genes ABC4, APOE, TLR4, and FBLN5 as being associated with susceptibility to AMD. However, the fraction of AMD patients carrying sequence changes in these genes was very small (Scholl et al 2007).

As mentioned above, complement activation has been implicated in susceptibility to AMD, mainly through complement factor $\mathrm{H}$. It is synthesized mainly in the liver and released into the blood where it is transported to other tissues. However, high levels of complement factor $\mathrm{H}$ are detected in the retina-choroid interface (Mandal and Ayyagari 2006; Chen et al 2007). This expression increases with age (Mandal and Ayyagari 2006). In addition, it was suggested that the RPE synthesizes complement factor H locally (Hageman et al 2005). The RPE production of factor $H$ would increase the local concentration of complement regulators and provide protection to inappropriate complement activation at sites of infection and inflammation (Rodriguez et al 2004). This mechanism would be particularly important in the retina, as the complement factors supplied by the blood may be restricted through the blood-brain barrier (Mandal and Ayyagari 2006).

Several reports have shown that oxidative mechanisms constitute the initial stimulus that triggers apoptosis, thereby contributing to the progression of AMD. The retina is highly susceptible to photo-oxidative damage due to its high oxygen demand, life-long exposure to light and the presence of polyunsaturated fatty acids highly enriched in the photoreceptor outer segments (Beatty et al 2000; Roth et al 2004). This scenario is aggravated with age, for there is a reduction in the local antioxidative enzymes in the RPE and a decrease in macular pigment density, which serves not only as a filter for short-wavelength light, but also as an antioxidant, through its two constituents, lutein and zeaxanthin. The age-related increase in oxidative stress leads to cellular events which in turn induce the histopathological changes associated with AMD, as described above. An animal model recently described the connection between oxidation, inflammation and pathology of AMD. In this model, mice were injected with mouse serum albumin adducted with carboxyethylpyrrole, an oxidation fragment generated from the protoreceptorenriched lipid docosahexaenoic acid. Injected mice develop antibodies to this hapten, fix high amounts of complement component 3 in Bruch's membrane, accumulate drusen below the RPE, and develop lesions in the RPE-photoreceptor interface that mimic AMD (Hollyfield et al 2007). The severity of the cellular lesions correlated with the antibody production titer.

\section{Outlook}

The aged RPE is characterized by several structural changes, which are exacerbated in AMD. These structural changes are known to be associated with an increase in oxidative stress and general decline of basic functions. Recently, it became evident that RPE and choriocapillaris express many if not all of the components and regulators of the complement cascade. Moreover, the RPE also plays an important role in the development of immune and inflammatory responses in the posterior part of the eye through production of cytokines. However, little is known about it in aging. The connection between oxidation and immune system in aging will provide a powerful approach for the elucidation of the many senile degenerative macular and peripheral retinal diseases such as AMD.

\section{Acknowledgments}

The author thanks Mary E Rayborn for critical review of the manuscript and for the help with electron microscopy and Joe G Hollyfield for critical comments on the manuscript and constant support. Human eyes used in this review were obtained through the Retinitis Pigmentosa Foundation Donor Program (Owings Mills, Maryland USA). Research was supported by NIH grants R21EY017153; a Research Center grant from the Foundation Fighting Blindness; a Challenge Grant from Research to Prevent Blindness; and an NEI infrastructure grant (EY015638).

\section{References}

Anderson DH, Mullins RF, Hageman GS, et al. 2002. A role for local inflammation in the formation of drusen in the aging eye. Am J Ophthalmol, 134:411-30.

Arnold JJ, Quaranta M, Soubrane G, et al. 1997. Indocyanine green angiography of drusen. Am J Ophthalmol, 124:344-56.

Bando H, Shadrach KG, Rayborn ME, et al. 2007. Clathrin and adaptin accumulation in drusen, Bruch's membrane and choroid in AMD and non-AMD donor eyes. Exp Eye Res, 84:135-42.

Baynes JW. 2001. The role of AGEs in aging: causation or correlation. Exp Gerontol, 36:1527-37.

Beatty S, Koh H-H, Phil M, et al. 2000. The role of oxidative stress in the pathogenesis of age-related macular degeneration. Surv Ophthalmol, 45:115-34.

Berman ER, Schwell H, Feeney L. 1974. The retinal pigment epithelium. chemical composition and structure. Invest Ophthalmol Vis Sci, 13:675-87.

Bird AC, Marshall J. 1986. Retinal pigment epithelial detachments in the elderly. Trans Ophthalmol Soc U K, 105:674-82.

Bok D. 1993. The retinal pigment epithelium: a versatile partner in vision. J Cell Sci Suppl, 17:189-95. 
Bonilha VL, Bhattacharya SK, West KA, et al. 2004. Proteomic characterization of isolated retinal pigment epithelium microvilli. $\mathrm{Mol}$ Cell Proteomics, 11:1119-27.

Boulton M, Dayhaw-Barker P. 2001. The role of the retinal pigment epithelium: topographical variation and ageing changes. Eye, 15:384-9.

Boulton M, Docchio F, Dayhaw-Barker P, et al. 1990. Age-related changes in the morphology, absorption and fluorescence of melanosomes and lipofuscin granules of the retinal pigment epithelium. Vision Res, 30:1291-303.

Boulton M, Dontsov A, Jarvis-Evans J, et al. 1993. Lipofuscin is a photoinducible free radical generator. $J$ Photochem Photobiol B, 19:201-4.

Chen M, Forrester JV, Xu H. 2007. Synthesis of complement factor H by retinal pigment epithelial cells is down-regulated by oxidized photoreceptor outer segments. Exp Eye Res, 84:635-45.

Chi A, Valencia JC, Hu ZZ, et al. 2006. Proteomic and bioinformatic characterization of the biogenesis and function of melanosomes. J Proteome Res, 5:3135-44.

Coursin DB, Cihla HP, Oberley TD, et al. 1992. Immunolocalization of antioxidant enzymes and isozymes of glutathione S-transferase in normal rat lung. Am J Physiol, 263:L679-91.

Cousins SW, Espinosa-Heidmann DG, Alexandridou A, et al. 2002. The role of aging, high fat diet and blue light exposure in an experimental model for basal laminar deposit formation. Exp Eye Res, 75:543-53. Erratum in: Exp Eye Res, 2003. 76:517.

Crabb JW, Miyagi M, Gu X, et al. 2002. Drusen proteome analysis: an approach to the etiology of age-related macular degeneration. Proc Natl Acad Sci USA, 99:14682-7.

Cruickshanks KJ, Klein R, Klein BE. 1993. Sunlight and age-related macular degeneration. The Beaver Dam Eye Study. Arch Ophthalmol, 111:514-18.

Davies SJ, D'Sousa R, Philips H, et al. 1993. Localisation of alpha, mu and pi class glutathione S-transferases in kidney: comparison with $\mathrm{CuZn}$ superoxide dismutase. Biochim Biophys Acta, 1157:204-8.

Davis WL, Matthews JL, Shibata K, et al. 1989. The immunocytochemical localization of superoxide dismutase in the enterocytes of the avian intestine: the effect of vitamin D3. Histochem J, 21:194-202.

Del Piore LV, Kuo Y-H, Tezel TH. 2002. Age-related changes in human RPE cell density and apoptosis. Invest Ophthalmol Vis Sci, 43:3312-18.

Del Viva MM, Agostini R. 2007. Visual spatial integration in the elderly. Invest Ophthalmol Vis Sci, 48:2940-6.

Dewan A, Liu M, Hartman S, et al. 2006. HTRA1 promoter polymorphism in wet age-related macular degeneration. Science, 314:989-92.

Dorey CK, Wu G, Eberstein D, et al. 1989. Cell loss in the aging retina: relationship to lipofuscin accumulation and macular degeneration. Invest Ophthalmol Vis Sci, 30:1691-9.

Dunaief JL, Dentchev T, Ying G-S, et al. 2002.The role of apoptosis in agerelated macular degeneration. Arch Ophthalmol, 120:1435-42.

Edwards AO, Ritter R III, Abel KJ, et al. 2005. Complement factor $\mathrm{H}$ polymorphism and age-related macular degeneration. Science, 308:421-4.

Eldred GE, Lasky MR. 1993. Retinal age pigments generated by selfassembling lysosomotrophic detergents. Nature, 361:724-6.

Farboud B, Aotaki-Kee A, Miyata et al. 1999. Development of a polyclonal antibody with broad epitope specificity for advanced glycation endproducts and localization of these epitopes in Bruch's membrane of the aging eye. Mol Vis, 5:11.

Feeney L. 1978. Lipofuscin and melanin of human retinal pigment epithelium. Fluorescence, enzyme cytochemical and ultrastructural studies. Invest Ophthalmol Vis Sci, 17:583-600.

Feeney-Burns L, Gao CL, Tidwell M. 1987. Lysosomal enzyme cytochemistry of human RPE, Bruch's membrane and drusen. Invest Ophthalmol Vis Sci, 28:1138-47.

Feeney-Burns L, Hilderbrand ES, Eldridge S. 1984. Aging human RPE: Morphometric analysis of macular, equatorial, and peripheral cells. Invest Ophthalmol Vis Sci, 25:195-200.

Gao H, Hollyfield JG. 1992. Aging of the human retina: differential loss of neurons and retinal pigment epithelial cells. Invest Ophthalmol Vis Sci, 33:1-17.
Gaillard ER, Atherton SJ, Eldred G, et al. 1995. Photophysical studies on human retinal lipofuscin. Photochem Photobiol, 61:448-53.

Garner A, Sarks S, Sarks JP. 1994. Degenerative and related disorders of the retina and choroid. In: Garner A, and Klintworth GK ed. Pathobiology of ocular diseases: a dynamic approach, 2nd ed, New York, NY: Marcel Dekker. pp. 631-74.

Glenn JV, Beattie JR, Barret L, et al. 2007. Confocal raman microscopy can quantify advanced glycation end product (AGE) modifications in Bruch's membrane leading to accurate, nondestructive prediction of ocular aging. Faseb J, 21:1-11.

Gold B, Merriam JE, Zernant J, et al. 2006. Variation in factor B (BF) and complement component $2(\mathrm{C} 2)$ genes is associated with age-related macular degeneration. Nat Genet, 38:458-62.

Green RW, Enger C. 1993. Age-related macular degeneration histopathologic studies. The 1992 Lorenz E. Zimmerman Lecture. Ophthalmology, 100:1519-34.

Hageman GS, Anderson DH, Johnson LV, et al. 2005. A common haplotype in the complement regulatory gene factor $\mathrm{H}(\mathrm{HF} 1 / \mathrm{CFH})$ predisposes individuals to age-related macular degeneration. Proc Natl Acad Sci USA, 102:7227-32.

Hageman GS, Hancox LS, Taibr AJ, et al. 2006. Extended haplotypes in the complement factor $\mathrm{H}(\mathrm{CFH})$ and $\mathrm{CFH}-$ related (CFHR) family of genes protect against age-related macular degeneration: characterization, ethnic distribution and evolutionary implications. Ann Med, 38:592-604.

Hageman GS, Luthert PJ, Victor Chong NH, et al. 2001. An integrated hypothesis that considers drusen as a biomarker of immune-mediated processes at the RPE-Bruch's membrane interface in aging and agerelated macular degeneration. Prog Ret Eye Res, 20:705-32.

Haines JL, Hauser MA, Schmidt S, et al. 2005. Complement factor H variant increases risk of age-related macular degeneration. Science, 308:419-21

Han M, Giese G, Schmitz-Valckenberg S, et al. 2007. Age-related structural abnormalities in the human retina-choroid complex revealed by two-photon excited autofluorescence imaging. J Bio Optics, 12:024012-1-7.

Handa JT, Verzijl N, Matsunaga H, et al. 1999. Increase in the advanced glycation end product pentosidine in Bruch's membrane with age. Invest Ophthalmol Vis Sci, 40:775-9.

Harman AM, Fleming PA, Hoskins RV, et al. 1997. Development and aging of cell topography in the human retinal pigment epithelium. Invest Ophthalmol Vis Sci, 38:2016-26.

Hinton DR, He S, Lopez PF. 1998. Apoptosis in surgically excised choroidal neovascular membranes in age-related macular degeneration. Arch Ophthalmol, 116:203-9.

Hogan MJ. 1972. Role of the retinal pigment epithelium in macular disease. Trans Am Acad Ophthalmol Otolaryngol, 76:64-80.

Hogan MJ. and Alvarado J. 1967. Studies on the human macula. IV. Aging changes in Bruch's membrane. Arch Ophthalmol, 77:410-20.

Hollyfield JG, Rayborn ME, Yang X, et al. 2007. Identification of an inflammatory signal from the outer retina causing age-related macular degeneration [abstract]. ARVO, 2356.

Jakobsdottir J, Conley YP, Weeks DE, et al. 2005. Susceptibility genes for age-related maculopathy on chromosome 10q26. Am J Hum Genet, 77:389-407.

Johnson LV, Leiter WP, Staples MK, et al. 2001. Complement activation and inflammatory response in drusen formation and age-related macular degeneration. Exp Eye Res, 73:887-96.

Katz ML, Drea CM, Eldred GE, et al. 1986. Influence of early photoreceptor degeneration on lipofuscin in the retinal pigment epithelium. Exp Eye Res, 43:561-73.

Katz ML, Robison WG Jr. 1984. Age-related changes in the retinal pigment epithelium of pigmented rats. Exp Eye Res, 38:137-51.

Klaver CC, Allikmets R. 2003. Genetics of macular dystrophies and implications for age-related macular degeneration. In: Wissinger B, Kohl S, Langenbeck U eds. Genetics in Ophthalmology. Dev Ophthalmol, Basel, Karger, 37:155-69. 
Kelin R, Peto T, Bird A, et al. 2004. The epidemiology of age-related macular degeneration. Am J Ophthalmol, 137:486-95.

Kelin R, Wang Q, Klein BEK, et al. 1995. The relationship of age-related maculopathy, cataract, and glaucoma to visual acuity. Invest Ophthalmol Vis Sci, 36:182-91.

Klein RJ, Zeiss C, Chew EY, et al. 2005. Complement factor H polymorphism in age-related macular degeneration. Science, 308:385-9.

Kohen R, Nyska A. 2002. Oxidation of biological systems: oxidative stress phenomena, antioxidants, redox reactions, and methods for their quantification. Toxicol Pathol, 30:620-50.

Kregel KC, Zhang HJ. 2007. An integrated view of oxidative stress in aging: basic mechanisms, functional effects, and pathological considerations. AM J Physiol Regul Integr Comp Physiol, 292:R18-36.

Kubasik-Juraniec J, Kmieć Z, Tukaj C, et al. 2004. The effect of fasting and refeeding on the ultrastructure of the hypothalamic paraventricular nucleus in young and old rats. Folia Morphol (Warsz), 63:25-35.

Lai YL, Rana MW. 1986. A study of photoreceptor-retinal pigment epithelium complex: age-related changes in monkeys. Proc Soc Exp Biol Med, 181:371-81.

Leibowitz HM, Krueger DE, Maunder LR, et al. 1980. The Framingham Eye Study monograph: An ophthalmological and epidemiological study of cataract, glaucoma, diabetic retinopathy, macular degeneration, and visual acuity in a general population of 2631 adults, 1973-1975. Surv Ophthalmol, 24:335-610.

Leung IY-F, Sandstrom MM, Zucker CL, et al. 2004. Nutrional manipulation of primate retinas, II: Effects of age, n-3 fatty acids, lutein, and zeaxanthin on retinal pigment epithelium. Invest Ophthalmol Vis Sci, 45:3244-56.

Mandal MN, Ayyagari R. 2006. Complement factor H: spatial and temporal expression and localization in the eye. Invest Ophthalmol Vis Sci, 47:4091-7.

Marshall J, Hussain AA, Starita C, et al. 1998. Aging and Bruch's membrane In: Marmor MF, Wolfensberger TJ ed. The Retinal Pigment Epithelium. New York, NY: Oxford University Press, pp. 669-92.

McFarland RA, Domey RG, Warren AB, et al. 1960. Dark adaptation as a fuction of age: I. Statistical analysis. J Gerontol, 15:149-54.

Morales E, Horn R, Pastor LM, et al. 2004. Involution of seminiferous tubules in aged hamsters: an ultrastructural, immunohistochemical and quantitative morphological study. Histol Histopathol, 19:445-55.

Mullins R, Aptsiauri N, Hageman GS. 2000. Dendritic cells and proteins associated with immune-mediated processes are associated with drusen and may play a central role in drusen biogenesis. Invest Ophthalmol Vis Sci, 41:S24.

Muse KE, Oberley TD, Sempf JM, et al. 1994. Immunolocalization of antioxidant enzymes in adult hamster kidney. Histochem $J, 26: 734-53$.

Nadon N. 2006. Exploiting the rodent model for studies on the pharmacology of lifespan extension. Aging Cell, 5:9-15.

Nakata K, Crabb JW, Holyyfield JG. 2005. Crystallin distribution in Bruch's membrane-choroid complex from AMD and age-matched donor eyes. Exp Eye Res, 80:821-6.

Nowak JZ. 2006. Age-related macular degeneration (AMD): pathogenesis and theraphy. Pharmacol Rep, 58:353-63.

Ohta Y, Kato H. 1975. Colour perception changes with age. Mod Prob Ophthalmol, 17:345.

Okubo A, Rosa RH Jr, Bunce CV, et al. 1999. The relashionships of age changes in retinal pigment epithelium and Bruch's membrane. Invest Ophthalmol Vis Sci, 40:443-9.

Page JW, Crognale MA. 2005. Differential aging of chromatic and achromatic visual pathways: behavior and electrophysiology. Vis Res, 45:1481-9.

Panda-Jones S, Jonas JB, Jakobczyk-Zmija M. 1996. Retinal pigment epithelial cell count, distribution, and correlations in normal human eyes. Am J Ophthalmol, 121:181-9.

Pauleikhoff D, Harper CA, Marshall, et al. 1990. Aging changes in Bruch's membrane. A histochemical and morphologic study. Ophthalmology, 97:171-8.
Pauleikhoff D, Zuels S, Sheraidah GS, et al. 1992. Correlation between biochemical composition and fluorescein binding of deposits in Bruch's membrane. Ophthalmology, 99:1548-53.

Renganathan K, Ng KP, Davies M, et al. 2007. Does lipofuscin contain protein? amino acid, protein and ultrastructural analysis of human lipofuscin [abstract]. ARVO, 5059 .

Rakoczy PE, Zhang D, Robertson T, et al. 2002. Progressive age-related changes similar to age-related macular degeneration in a transgenic mouse model. Am J Pathol, 161:1515-24.

Rayborn ME, Sakagushi H, Shadrach KG, et al. 2006. Annexins in Bruch's membrane and drusen. Adv Exp Med Biol, 572:75-8.

Rayborn ME, Hollyfield JG, Bonilha VL. 2005. Age-related changes in the retinal pigment epithelium apical surface [abstract]. ARVO, 3049.

River A, Fisher SA, Fritscher LG, et al. 2005. Hypothetical LOC387715 is a second major susceptibility gene for age-related macular degeneration, contributing independently of complement factor $\mathrm{H}$ to disease risk. Hum Mol Genet, 14:3227-36.

Rodriguez DC, Esparza-Gordillo J, Goicoechea DJ, et al. 2004. The human complement factor $\mathrm{H}$ : functional roles, genetic variations and disease associations. Mol Immunol, 41:355-67.

Roth F, Bindewald A, Holz FG. 2004. Key pathophysiologic pathways in age-related macular disease. Graefe's Arch Clin Exp Ophthalmol, 242:710-16

Rozanowska M, Jarvis-Evans J, Korytowski W, et al. 1995. Blue lightinduced reactivity of retinal age pigment. In vitro generation of oxygenreactive species. $J$ Biol Chem, 270:18825-30.

Rozanowska M, Wessels J, Boulton M, et al. 1998. Blue light-induced singlet oxygen generation by retinal lipofuscin in non-polar media. Free Rad Biol Med, 24:1107-12.

Sarks SH. 1976. Aging and degeneration in the macular region: a clinicopathological study. Br J Ophthalmol, 60:324-41.

Sarks JP, Sarks SH, Killingsworth MC. 1988. Evolution of geographic atrophy of the retinal pigment epithelium. Evolution of geographic atrophy of the retinal pigment epithelium. Eye, 2:552-77.

Sarna T, Burke JM, Korytowski W, et al. 2003. Loss of melanin from human RPE with aging: possible role of melanin photooxidation. Exp Eye Res, 76:89-98.

Schmucker DL, Sachs H. 2002. Quantifying dense bodies and lipofuscin during aging: a morphologist's perspective. Arch Gerontol Geriatr, 34:249-61.

School HPN, Fleckenstein M, Issa PC, et al. 2007. An update on the genetics of age-related macular degeneration. Mol Vis, 13:196-0205.

Schutt F, Davies S, Kopitz J, et al. 2001. Photodamage to human RPE cells by A2-E, a retinoid component of lipofuscin. Invest Ophthalmol Vis Sci, 41:2303-8.

Schutt F, Bergmann M, Holz FG, et al. 2002. Isolation of intact lysosomes from human RPE cells and effects of A2-E on the integrity of the lusosomal and other cellular membranes. Graefes Arch Clin Exp Ophthalmol, 240:983-8.

Sheraidah G, Steinmetz R, Maguire J, et al.1993. Correlation between lipids extracted from Bruch's membrane and age. Ophthalmology, 100:47-51.

Spencer KL, Hauser MA, Olson LM, et al. 2007. Protective effect of complement factor B and complement component 2 variants in age-related macular degeneration. Hum Mol Genet, 16:1986-92.

Sunness JS. 1999. The natural history of geographic atrophy, the advanced atrophic form of age-related macular degeneration. Mol Vis, 5:25.

Suter M, Reme C, Grimm C, et al. 2000. Age-related macular degeneration. The lipofuscin component N-retinyl-N-retinylidene ethanolamine detaches proapoptotic proteins from the mitochondria and induces apoptosis in mammalian retinal pigment epithelial cells. $J$ Biol Chem, 275:39625-30.

Streeten BW. 1969. Development of the human retinal pigment epithelium and the posterior segment. Arch Ophthalmol, 81:383-94.

Terman A, Gustafsson B, Brunk UT. 2007. Autophagy, organelles and ageing. J Pathol, 211:134-43. 
Tso MOM, Friedman E. 1968. The retinal pigment epithelium: III. Growth and development. Arch Ophthalmol, 80:214-16.

Ugarte M, Hussain AA, Marshall J. 2006. An experimental study of the elastic properties of the human Bruch's membrane-choroid complex: relevance to ageing. Br J Ophthalm, 90:621-6.

Van Remmen H, Hamilton ML, Richardson A. 2003. Oxidative damage to DNA and aging. Exerc Sport Sci Rev, 31:149-53.

Wang Z, Dillon J, Gaillard ER. 2006. Antioxidant properties of melanin in retinal pigment epithelial cells. Photochem Photobiol, 82:474-9.

Warrburton S, Davis WE, Southwick K, et al. 2007. Proteomic and phototoxic characterization of melanolipofuscin: correlation to disease and model for its origin. Mol Vis, 13:318-29.

Wassell J, Davies S, Bardsley W, et al. 1999. The photo-reactivity of the retinal age pigment lipofuscin. $J$ Biol Chem, 274:23828-32.

Watzke RC, Soldevilla JD, Trune DR. 1993. Morphometric analysis of human retinal pigment epithelium: correlation with age and location. Curr Eye Res, 12:133-42.

Weale RA. 1975. Senile changes in visual acuity. Trans Ophthalmol Soc UK, 95:36-8.

Weisse I. 1995. Changes in the aging rat retina. Ophthalmic Res, 27:154-63.
Werner JS. 2005. Night vision in the elderly: consequences for seeing through a "blue filtering" intraocular lens. Br J Ophthalmol, 89:1518-21.

Wing GL, Blanchard GC, Weiter JJ. 1978. The topography and age relationship of lipofuscin concentration in the retinal pigment epithelium. Invest Ophthalmol Vis Sci, 17:601-7.

Xu GZ, Li WW, Tso MOM. 1996. Apoptosis in human retinal degenerations. Trans Am Ophthalmol Soc, 94:411-31.

Yang Z, Cammp NJ, Sun H, et al. 2006. A variant of the HTRA1 gene increases susceptibility to age-related macular degeneration. Science, 314:992-3.

Yasukawa T, Wiedemann P, Hoffman S, et al. 2007. Glycoxidized particles mimic lipofuscin accumulation in aging eyes: a new age-related macular degeneration model in rabbits. Graefes Arch Clin Exp Ophthalmol, 245:1475-85.

Young RW. 1987. Pathophysiology of age-related macular degeneration. Surv Ophthalmol, 31:291-306.

Zarbin MA. 2004. Current concepts in the pathogenesis of age-related macular degeneration. Arch Ophthalmol, 122:598-614.

Zinn KM, Benjamin-Henkind JV. 1979. Anatomy of the human retinal pigment epithelium. In: Zinn KM and Marmor MF ed. The Retinal Pigment Epithelium. Cambridge, MA: Harvard University Press, pp. 3-31. 